\section{LOS CRITERIOS MICROBIOLÓGICOS: PRINCIPIOS PARA SU ESTABLECIMIENTO Y APLICACIÓN EN LA SEGURIDAD ALIMENTARIA}

\author{
Antonio Valero Díaz \\ Universidad de Córdoba \\ ORCID iD: https://orcid.org/0000-0001-8597-0740
}

avalero@uco.es

Cómo citar este artículo/Citation: Valero Díaz, A. (2020). Los criterios microbiológicos: principios para su establecimiento y aplicación en la seguridad alimentaria. Arbor, 196 (795): a537. https://doi.org/10.3989/arbor.2020.795n1001

Recibido: 26 febrero 2019. Aceptado: 29 octubre 2019.

RESUMEN: La legislación en materia de seguridad alimentaria ha ido encaminada hacia una mayor estandarización de las producciones que, junto con las certificaciones de calidad existentes, tienen como objetivo un incremento del nivel de protección de la salud pública. Está constatada la necesidad de que la industria agroalimentaria disponga de herramientas con las que poder armonizar sus producciones y gestionar adecuadamente sus sistemas de calidad para ofrezcer una mayor confianza a los consumidores finales. La implementación de los criterios microbiológicos está enfocada a facilitar dicha armonización posibilitando la discriminación de lotes defectuosos y actuando como herramientas de control en las industrias. Por tanto, el conocimiento de los principios, componentes y factores que influyen sobre la eficiencia de los criterios microbiológicos ayuda a un mejor entendimiento de las consecuencias de su aplicación. En el presente artículo se abordan los principales fundamentos, metodologías y aplicaciones de los criterios microbiológicos en alimentos de cara a su implementación en los sistemas de calidad de las industrias agroalimentarias. Asimismo, se discuten algunos aspectos sobre las posibles limitaciones y repercusiones sobre la seguridad alimentaria.

PALABRAS CLAVE: contaminación microbiana; métricas del riesgo; planes de muestreo; gestión del riesgo; límite microbiológico; probabilidad de aceptación.

\section{MICROBIOLOGICAL CRITERIA: PRINCIPLES FOR THEIR ESTABLISHMENT AND APPLICATION IN FOOD SAFETY}

Copyright: (C) 2020 CSIC. Este es un artículo de acceso abierto distribuido bajo los términos de la licencia de uso y distribución Creative Commons Reconocimiento 4.0 Internacional (CC BY 4.0).

ABSTRACT: Legislation on food safety has led to the standardisation of food production which, together with existing quality certifications, aim to increase the level for protection of public health. The need is recognized for the agri-food industry to have tools to harmonize their production and to adequately manage their quality systems in order to increase consumers' confidence. The implementation of microbiological criteria is focused on facilitating this harmonization by enabling differentiation of defective lots and acting as control tools at the industrial level. Therefore, knowledge on the principles, components and factors influencing the efficiency of microbiological criteria may be helpful to better understand the consequences of their application. In the present paper the main principles, methodologies and applications of microbiological criteria in foods are addressed for their implementation as part of management quality systems in agri-food industries. In addition, potential limitations and impacts of microbiological criteria on food safety are discussed.

KEYWORDS: microbial contamination, risk-based metrics, sampling plans; risk management; microbiological limit; acceptance probability. 


\section{INTRODUCCIÓN. PERSPECTIVAS E IMPLICACIONES DEL CONTROL DE LA CALIDAD EN INDUSTRIAS AGRO- ALIMENTARIAS}

La industria agroalimentaria ha sufrido en los últimos años sucesivas modificaciones en los procesos de producción con objeto de satisfacer las demandas de los consumidores hacia alimentos más saludables, nutritivos, inocuos y apetecibles. El incremento de la producción de alimentos está gobernado por un notable avance científico y tecnológico, mejora genética, desarrollo de compuestos fertilizantes y pesticidas o uso de antibióticos y sustancias promotoras del crecimiento en alimentos de origen animal. Esta sucesiva introducción de elementos de origen humano ha coexistido en los últimos tiempos con métodos de producción más sostenibles conducentes a garantizar la eficiencia energética, la mejora de las condiciones medioambientales o la reducción de la generación de residuos, entre otros fines.

La utilización de enfoques de tipo preventivo facilita la implementación de las medidas de control a lo largo de la cadena de producción y el desarrollo de sistemas de respuesta rápida, que permitan prever una correcta toma de decisiones. El análisis de riesgos va en consonancia con los enfoques preventivos, siendo el procedimiento científico que sirve para priorizar las políticas de gestión de la calidad e inocuidad alimentaria y establecimiento de los objetivos de salud pública (Commission Regulation (EC) No 178/2002). La evaluación de riesgos es el componente científico que se deriva del análisis de riesgos y consiste en un procedimiento sistematizado que se lleva a cabo con el fin de conocer la naturaleza, magnitud y probabilidad que presenta un peligro potencialmente presente en un alimento. Los resultados derivados de una evaluación de riesgos constituyen una herramienta valiosa para la toma de decisiones, la detección de puntos críticos en la cadena agroalimentaria, la evaluación de estrategias de mitigación y la elaboración de normativas o estándares que faciliten el comercio de alimentos (Word Health Organization / Food and Agriculture Organization of the Unites Nations, 2006).

Una de las herramientas más valiosas que tienen las industrias agroalimentarias son los sistemas de gestión de la calidad e inocuidad alimentaria (GCIA), los cuales tienen su principal fundamento en la norma internacional ISO 22000, que constituye una herramienta eficaz para lograr alimentos inocuos y se antoja fundamental para la gestión empresarial. La norma ISO 22000 reúne los requisitos específicos para alcanzar la inocuidad de los alimentos a partir de los principios del Análisis de Peligros y Puntos de Control Críticos (APPCC), y propone aplicar este proceso a través de los conceptos de gestión de la calidad, basados en la norma internacional ISO 9001. Ambas normas pueden ser certificadas en forma individual; no obstante, pueden implementarse en forma integrada a través de la aplicación de ISO 22000.

La industria agroalimentaria posee herramientas basadas en la utilización de principios como las Buenas Prácticas de Higiene y Fabricación (BPH, BPF), los principios del APPCC y los sistemas de autocontrol (APPCC + Planes Generales de Higiene o Programas de Prerrequisitos). Estas herramientas ayudan a documentar el proceso y normalmente vienen acompañadas por sistemas de gestión de la trazabilidad, que permiten identificar puntos críticos a lo largo de la cadena de producción-consumo y establecer medidas correctivas (Commission Regulation (EC) No 852/2004). La armonización de estos sistemas se basa en la implementación de los criterios microbiológicos, que tienen como principal objetivo validar y verificar las medidas de control llevadas a cabo dentro de los sistemas GCIA para garantizar el suministro de alimentos seguros para el consumidor. Sin embargo, su aplicación no siempre es sencilla ni factible, por lo que es necesario un conocimiento profundo de los factores que influyen sobre su eficiencia, así como los posibles efectos beneficiosos y las limitaciones resultantes de su aplicación.

\section{LOS PLANES DE MUESTREO COMO HERRAMIENTA DE GESTIÓN DE LA SEGURIDAD ALIMENTARIA}

Los alimentos generalmente constituyen matrices complejas donde interaccionan múltiples factores de forma simultánea o diferida produciendo constantes modificaciones sobre el producto que muchas veces son difíciles de predecir. La integración de los enfoques preventivos destinados a abarcar la cadena "del campo a la mesa" deben inevitablemente contemplar distintos agentes, fases y operaciones, algunos de ellos no cuantificables ni predecibles, que complican en cierto modo la armonización de los procedimientos que componen un sistema GCIA.

A la hora de diseñar un sistema GCIA es necesario conocer las causas por las cuales un lote de producción puede estar contaminado, así como el tipo de peligro a tratar y el origen de dicha contaminación. Para ello, los llamados procedimientos de muestreo están orientados hacia la medición de una serie de parámetros de calidad y a la toma de porciones representativas de un lote determinado con objeto de detectar la presencia de contaminantes.

La mayoría de los procedimientos de muestreo comprenden la selección de una o varias muestras de un lote, la inspección o el análisis de las muestras y la clasificación del lote (como aceptable o no aceptable) a partir del resultado de la inspección o el análisis de la muestra (Codex Alimentarius Commission, 
CAC/GL 50-2004). Para ello, un plan de muestreo constituye un procedimiento normalizado mediante el cual se inspecciona y clasifica un lote. Básicamente el plan de muestreo contemplará el número de unidades seleccionadas, así como el número máximo de unidades del lote que no puede exceder un cierto nivel o límite ( $n$ y $c$, respectivamente). Según las definiciones otorgadas por el Codex Alimentarius, los planes de muestreo que involucren cambios se denominan esquemas de muestreo, mientras que el conjunto de planes y esquemas de muestreo se definen como sistemas de muestreo.

A su vez, los planes de muestreo se clasifican en planes de aceptación o atributos, que miden una cualidad determinada de un alimento con objeto de aceptar o rechazar un lote (por ej., presencia de Salmonella spp. en ovoproductos), mientras que los planes por variables miden un valor o concentración determinada en un alimento con objeto de comprobar si se ajusta o no a unas especificaciones determinadas (por ej., nivel de $\mathrm{Sn}$ en envases alimentarios).

En la práctica, los planes de muestreo por aceptación son los que generalmente se llevan a cabo en microbiología con objeto de detectar lotes contaminados con un suficiente nivel de confianza. En este sentido, es necesario saber que debe establecerse un margen de seguridad de forma que el plan de muestreo que se vaya a aplicar sea lo suficientemente efectivo como para poder ser utilizado como medida de control. El muestreo del producto final puede constituir una herramienta eficaz de control, o bien un procedimiento sujeto a total aleatoriedad donde los resultados positivos generalmente presentan una gran relevancia, mientras que los negativos no permiten tener una total garantía de ausencia de contaminación. A modo de ejemplo, un $1 \%$ de unidades contaminadas en un lote puede representar un porcentaje aparentemente pequeño en términos relativos, pero cuando lo relacionamos con un tamaño de lote suficientemente grande (por ej. 100.000 uds.) supondría un total de 1.000 uds. contaminadas, lo que supondría un grave problema de seguridad alimentaria en caso de que se pusieran a la venta.

Por ello, el principal objeto de un plan de muestreo consiste en incrementar al máximo la probabilidad de detección de lotes contaminados (probabilidad de rechazo) de forma que pueda tener un alto nivel de discriminación. Dicho de otro modo, es necesario tratar de reducir al máximo el porcentaje de unidades de un lote que puedan exceder un nivel especificado de contaminación (probabilidad de aceptación). En el caso de los alimentos, como entidades heterogéneas, la probabilidad de detección dependerá del tamaño de muestra, del nivel de contaminación y de la variabilidad de dicha contaminación en el lote.
Los microorganismos se definen como entidades discretas, es decir, como entes divisibles un número finito de veces. Esto hace que la contaminación microbiológica presente en los alimentos sea diferente en función del tipo de alimento y del peligro biológico de que se trate. En la mayoría de las ocasiones, los microorganismos están distribuidos de forma heterogénea y esto hace que el plan de muestreo sea un proceso cuyo comportamiento intrinsicamente no es determinista, sino que está motivado tanto por las acciones predecibles del proceso como por elementos aleatorios. En otras palabras, los planes de muestreo se definen como procesos estocásticos, altamente influenciados por la aleatoriedad de la contaminación presente en el alimento, como del sistema de muestreo adoptado para detectar dicha contaminación. En la figura 1 se muestran dos lotes con un nivel de contaminación real similar. Sin embargo, la propia aleatoriedad del muestreo hace que en algunos casos no se detecte contaminación, mientras que en otros sí, lo que supone que la contaminación asumida en los lotes pueda ser totalmente diferente a la real. A todo ello, hay que añadir que los métodos de análisis no siempre son capaces de detectar en un $100 \%$ la contaminación real presente en el lote, especialmente cuando alcanza niveles bajos, con lo que nos tenemos que preguntar irremediablemente si el muestreo es realmente útil para garantizar la seguridad alimentaria. La respuesta a esta pregunta no es absoluta, sino que tiene distintas vertientes en función de la situación o escenario que nos encontremos, con lo que no hay ni que desechar siempre ni que ceñirse solamente al muestreo como garantía del control de las producciones. En este sentido una gestión efectiva consistirá en utilizar las herramientas que tenemos a nuestro alcance para maximizar el control de la seguridad alimentaria de las producciones, y los planes de muestreo, así como los criterios microbiológicos, son solo algunas de ellas.

Figura 1. Definición del proceso estocástico de los planes de muestreo y su influencia sobre la detección de lotes contaminados

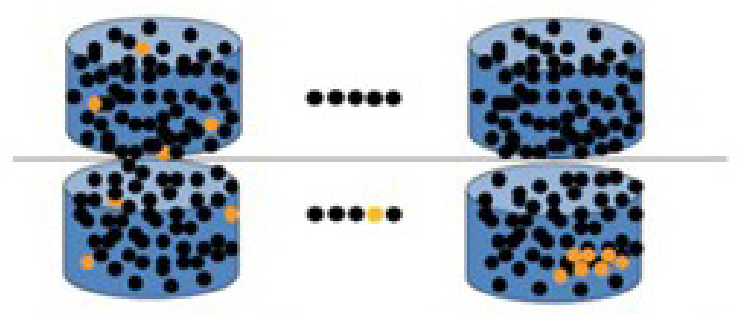

Contaminación real

Contaminación asumida presente en el lote presente en el lote

Fuente: elaboración propia. 
DEFINICIÓN, COMPONENTES Y PRINCIPIOS PARA LA APLICACIÓN DE LOS CRITERIOS MICROBIOLÓGICOS EN ALIMENTOS

Como se ha descrito anteriormente, la finalidad de la aplicación de los sistemas GCIA en microbiología es la de evitar que los productos alimenticios contengan microorganismos, toxinas o metabolitos en cantidades que supongan un riesgo inaceptable para la salud humana.

Con objeto de armonizar las medidas de protección de la salud pública, se necesita establecer criterios de seguridad alimentaria que indiquen la aceptabilidad de un lote con relación a la presencia de microorganismos patógenos. A este respecto, el informe relativo a los criterios microbiológicos aplicables a los productos alimenticios (Commission Regulation (EC) No 1441/2007) define criterio microbiológico como un criterio que determina la aceptabilidad de un producto, de un lote de productos alimenticios o de un proceso, basándose en la ausencia, presencia o número de microorganismos, y en la cantidad de sus toxinas/metabolitos por unidad de masa, de volumen, de superficie o lote.

A su vez, los criterios microbiológicos se dividen en:

Criterio de seguridad alimentaria: criterio que define la aceptabilidad de un producto o un lote de productos alimenticios y es aplicable a los productos comercializados (ej. Listeria monocytogenes en alimentos listos para el consumo o Ready-To-Eat);

Criterio de higiene de proceso: criterio que indica el funcionamiento aceptable del proceso de producción; este criterio, que no es aplicable a los productos comercializados, establece un valor de contaminación indicativo por encima del cual se requieren medidas correctoras para mantener la higiene del proceso conforme a la legislación alimentaria (por ej. enterobacterias en canales de porcino).

Los criterios microbiológicos se derivan de los planes de muestreo, aunque incluyen más información, tal como:

- Una descripción de los peligros biológicos y de las causas que suscitan la aparición de dichos peligros;

- Los métodos de análisis para su detección y cuantificación;

- Un plan de muestreo donde se definan el número de unidades y la magnitud de la unidad analítica;

- Los límites microbiológicos que se consideran apropiados para el alimento en el punto o puntos especificados de la cadena agroalimentaria;

- El número de unidades analíticas que deben ajustarse a esos límites.
- El alimento al que se aplica el criterio y el punto o los puntos de la cadena agroalimentaria en que se aplica el criterio;

- Las medidas a adoptar en caso de resultados insatisfactorios.

Recientemente, EFSA publicó una opinión acerca de la forma de interpretación de los criterios microbiológicos, así como de las herramientas y metodologías disponibles para llevarlos a cabo (EFSA Panel on Biological Hazards et al., 2017). Entre otros aspectos, se destacó el hecho diferenciador del papel que juegan los asesores del riesgo (encargados de evaluar el impacto de los criterios con respecto a la seguridad alimentaria), con relación a los gestores del riesgo (los cuales son los responsables de la toma de decisiones sobre la aplicación de los criterios).

Es importante tener en cuenta que los criterios microbiológicos no deben aplicarse siempre de forma sistemática y por defecto, sino que es imprescindible la evaluación del coste/beneficio a la hora de aplicarlos, en relación con la posibilidad de proporcionar al consumidor un alimento inocuo y apto para el consumo.

\section{METODOLOGÍAS PARA LA DERIVACIÓN DE CRITERIOS MICROBIOLÓGICOS EN ALIMENTOS}

De acuerdo con la legislación europea alimentaria vigente (Commission Regulation (EC) No 178/2002; Commission Regulation (EC) 852/2004; Commission Regulation (EC) No 1441/2007), la aplicación de los criterios microbiológicos está orientada hacia una armonización de los sistemas GCIA. Sin embargo, para una correcta interpretación de estos es preciso conocer cómo se pueden derivar mediante la recopilación de información sobre aspectos tales como:

- La distribución de la contaminación microbiana en un lote;

- La definición de los límites microbiológicos por encima de los cuales se considera el rechazo de lotes. En los planes de muestreo por atributos de dos y tres clases, dichos valores se corresponden con los límites $m$ (inferior) y $M$ (superior);

- Una descripción del método de análisis que permita conocer la probabilidad de detectar la presencia de contaminación, así como los límites de detección y cuantificación. En este sentido conviene saber que la eficiencia de los planes de muestreo y, por tanto, de los criterios microbiológicos viene marcada por los valores de sensibilidad (o proporción de muestras positivas que son realmente detectadas por el método analítico) y especificidad (o proporción de muestras negativas que son realmente detectadas por el método analítico); 
- El nivel de confianza que se desea implementar para determinar la no conformidad de un lote (por ej. probabilidad de rechazo $(\mathrm{Pr})=95 \%$; o probabilidad de aceptación $(\mathrm{Pa})=5 \%)$.

Muchos de estos puntos se pueden obtener a partir de información previa o datos históricos de los que se disponga, o bien realizando una serie de asunciones que permitan derivar un criterio microbiológico. En concreto, el conocimiento de la distribución de la contaminación microbiana en un lote es muchas veces difícil de predecir. No obstante, esta información es de alta relevancia a la hora de diseñar un adecuado sistema de muestreo de forma que se optimice la probabilidad de detección de muestras positivas o lotes defectuosos.

En una situación ideal, si partiendo de una matriz concreta pudiésemos visualizar espacialmente cómo están distribuidos los microorganismos, tendríamos un conocimiento completo acerca de la distribución de la contaminación en un lote. Sin embargo, esta información no está disponible en la mayoría de las ocasiones (a no ser que llevemos a cabo exhaustivos estudios en el nivel microscópico, lo cual lógicamente no es viable). En una situación intermedia, podríamos conocer el número de células que ocupan un espacio concreto del alimento, pero desafortunadamente tampoco existe mucha información al respecto, y además el tiempo requerido para obtenerla sería inasumible, sobre todo cuando es preciso tomar decisiones en un tiempo corto. Por tanto, para poder definir matemáticamente la disposición de la contaminación microbiana en un lote o alimento, tenemos que recurrir a distribuciones estadísticas (véase figura 2). Los parámetros que definen cada una de estas distribuciones podrán utilizarse para reflejar el grado de contaminación de un lote, así como la variabilidad asociada a la contaminación en ese lote. En este último caso, las distribuciones no son más que "modelos" que intentan reflejar la disposición de los microorganismos en un alimento, es decir, su nivel de concentración y la variabilidad de la misma en un lote o en distintos lotes.

Algunas de las distribuciones estadísticas más utilizadas para la derivación de criterios microbiológicos son la binomial, la normal, la Poisson o la binomial negativa. Los parámetros que se pueden utilizar para definir el nivel medio de contaminación de un lote y su variabilidad asociada son $\mu$ y $\sigma$, respectivamente. En la figura 3 se representan tres escenarios donde se muestra la contaminación microbiana en un alimento concreto y la distribución estadística que puede estar asociada a dicha contaminación.

Existen algunos estudios publicados sobre la importancia de las distribuciones estadísticas a la hora de derivar criterios microbiológicos (Schothorst, Zwietering, Ross, Buchanan y Cole, 2009). En muchos de ellos, la eficiencia de la implementación de los criterios microbiológicos se cuantifica gracias al conocimiento de las propiedades de la distribución normal. Siguiendo los principios establecidos por el teorema central del límite, cualquier distribución estadística se puede aproximar a una distribución normal a medida que se incrementa el tamaño de muestra, asumiendo que la media y varianza ( $\mu$ y $\sigma$ ) son independientes entre sí. Con ello, las distribuciones binomial y Poisson se aproximan a una normal a medida que el número de muestras tiende a infinito.

Gracias al conocimiento de estas propiedades se puede calcular de forma relativamente sencilla la eficiencia de los planes de muestreo y criterios microbiológicos en alimentos mediante las llamadas curvas características de operación (figura 4).

Figura 2. Representación de la distribución de la contaminación microbiana presente en un lote o alimento en función del nivel de información disponible

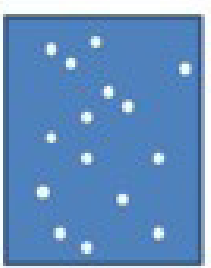

\begin{tabular}{|l|l|l|l|}
\hline 4 & 4 & 4 & 2 \\
\hline 0 & 0 & 1 & 3 \\
\hline 0 & 4 & 2 & 4 \\
\hline 2 & 1 & 1 & 0 \\
\hline
\end{tabular}
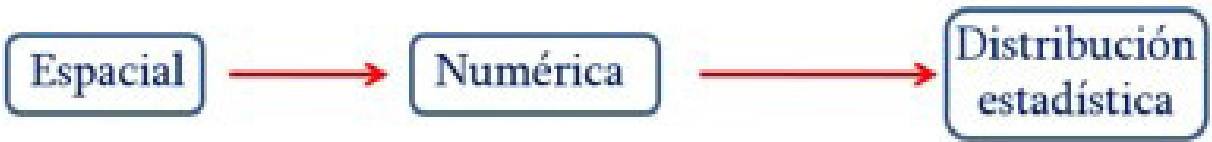
Figura 3. Representación de la distribución de la contaminación microbiana presente en un lote o alimento y la distribución estadística asociada

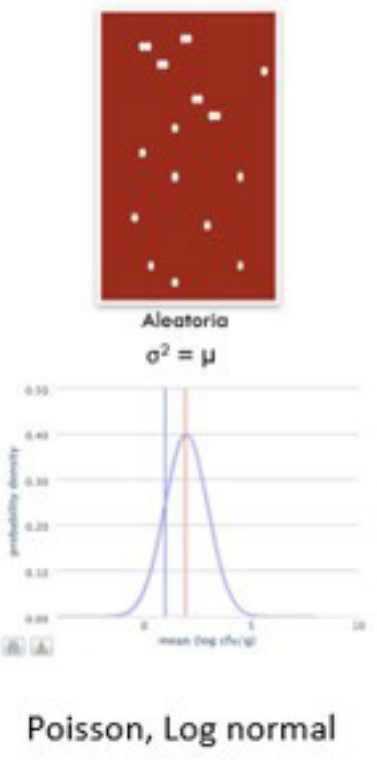

Poisson, Log normal

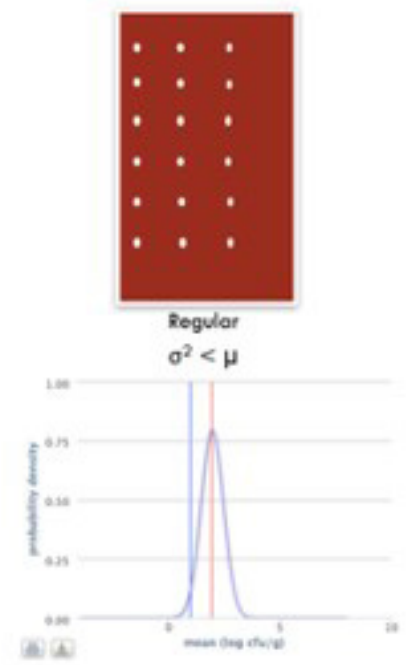

Log normal

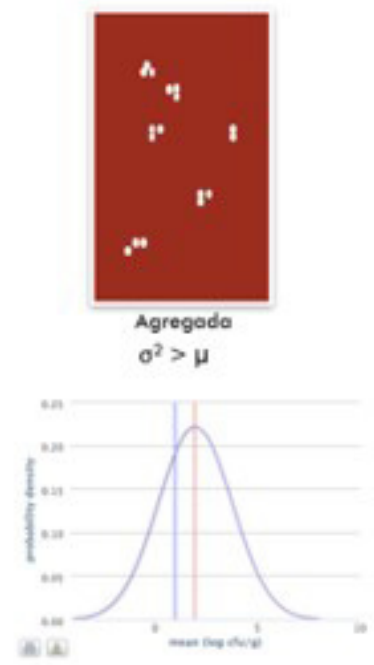

Binomial negativa, Poisson-

log normal

Fuente: elaboración propia.

Figura 4. Curvas características de operación que evalúan la eficiencia de la implementación de planes de muestreo y criterios microbiológicos en alimentos.

Figura 4a. Efecto del número de muestras (n) sobre la probabilidad de aceptación del lote ( $m=1$ log ufc/g).de muestreo y criterios microbiológicos en alimentos

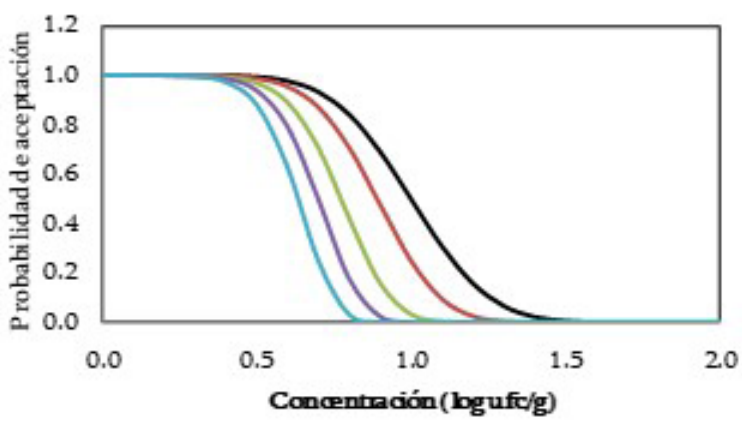

${ }^{\mathrm{n}}=1-\mathrm{n}=2-\mathrm{n}=5-\mathrm{n}=10 \quad \mathrm{n}=20$

Fuente: elaboración propia.

En la figura $4 a$, se pueden estimar los valores de concentración microbiana máximos admisibles en el lote de forma que la $\operatorname{Pr}=95 \%$. Se puede comprobar que a medida que se incrementa el número de muestras, para un valor de $m=1$ log ufc/g, los valores de concentración (log ufc/g) a los cuales $\mathrm{Pr}=95 \%$ son de $1.33(n=1) ; 1.15(n=2) ; 0.97(n=5) ; 0.87(n=10)$ y 0.78 $(n=10)$. Por tanto, conforme se va incrementando el número de muestras existe una mayor probabilidad
Figura 4b. Efecto de la variabilidad de la contaminación del lote (SD) sobre la probabilidad de aceptación ( $m=1$ $\log$ ufc/g)

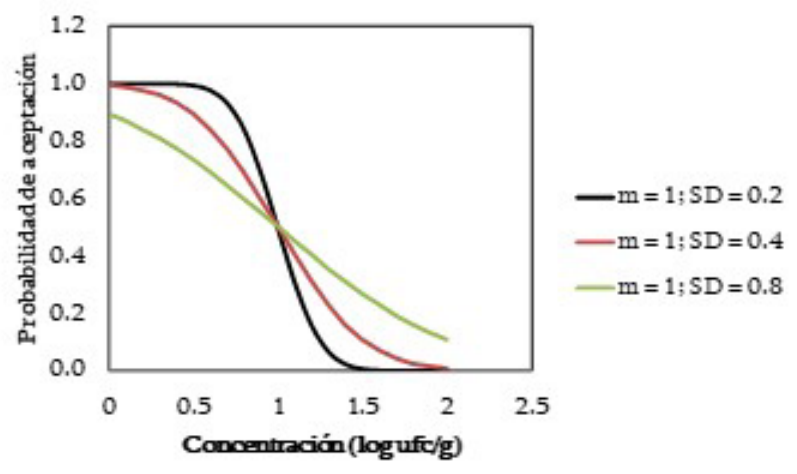

de detectar niveles más bajos de concentración, con lo que el criterio microbiológico será más estricto.

Por otra parte, en la figura $4 \mathrm{~b}$ se representa la influencia de los valores de desviación estándar (SD) sobre la eficiencia de la implementación de los criterios microbiológicos. La desviación estándar es una medida que refleja el grado de variabilidad de la contaminación microbiana presente en el lote. A medida que aumenta el valor de SD, el criterio microbio- 
lógico es menos restrictivo, teniendo unos valores máximos admisibles de concentración (log ufc/g) a los cuales $\operatorname{Pr}=95 \%$ de $1.33(\mathrm{SD}=0.2) ; 1.66(\mathrm{SD}=0.4)$ y $2.31(S D=0.8)$.

De esta forma, gracias a las distribuciones estadísticas y al conocimiento de los parámetros principales asociados se puede evaluar la influencia de la implementación de criterios microbiológicos en alimentos. Obviamente, en muchos casos esta situación no es tan trivial, sino que deben tenerse en cuenta las variables asociadas que influyen en el proceso de elaboración, el tipo de peligro biológico y la matriz al cual está asociado y, como se ha comentado antes, la ratio coste/beneficio resultante de la aplicación del criterio microbiológico.

\section{ESTABLECIMIENTO DE CRITERIOS MICROBIOLÓGICOS BASADOS EN MÉTRICAS DEL RIESGO}

La gestión de la seguridad microbiológica mediante enfoques basados en el riesgo ha provocado un cambio sustancial en la adopción de metodologías y controles por parte de las industrias alimentarias a lo largo de la cadena de producción (Codex Alimentarius Commission CAC/GL 62-2007). Dichos enfoques han promovido un cambio de estrategias de control basadas en la aparición de peligros en el producto final hacia el establecimiento de medidas de gestión del riesgo siguiendo las recomendaciones planteadas por organizaciones internacionales como EFSA, FAO/OMS o el Codex Alimentarius. Esto requiere disponer de herramientas estadísticas e informáticas que intenten predecir el comportamiento de los microorganismos durante la cadena de producción-consumo, así como la necesidad de armonizar criterios microbiológicos basados en medidas de gestión del riesgo.

La gestión del riesgo es un proceso dentro del análisis de riesgos que trata de establecer medidas de control adecuadas para la mejora de la calidad y de la seguridad alimentaria, en base a los resultados de una evaluación del riesgo. Así pues, el gestor del riesgo es una organización gubernamental nacional o internacional encargada de la gestión del riesgo microbiológico.

Una forma de relacionar los requerimientos de los programas de seguridad alimentaria y su impacto sobre la salud pública es mediante el desarrollo de herramientas tales como objetivos de seguridad alimentaria (FSO) y objetivos de rendimiento (PO). Estos objetivos, conocidos como métricas del riesgo, están relacionados con el nivel de riesgo que una sociedad está inclinándose a aceptar (nivel adecuado de protección o ALOP). Un FSO es "la frecuencia máxima y la concentración máxima de un peligro en un alimento al momento del consumo, que provee o contribuye al nivel adecuado de protección (ALOP)" (ICMSF, 2002).
Un PO es en cambio la "frecuencia máxima y concentración de un peligro microbiológico en un alimento en una determinada etapa de la cadena alimentaria antes del consumo que contribuye a alcanzar el objetivo de seguridad alimentaria (FSO)".

Las métricas del riesgo están definidas por la International Commission on Microbiological Specifications for Foods (ICMSF) (2002) y han sido posteriormente aplicadas en varios estudios (Stringer, 2005; Valero, 2015; Zwietering, Gorris y Farber, 2015) como criterios de seguridad alimentaria para distintos patógenos. Las métricas del riesgo, al contrario que los criterios microbiológicos, no incluyen información acerca del alimento, plan de muestreo, límite microbiológico o método de análisis. Son, por tanto, niveles que se establecen en base a unos requisitos de seguridad alimentaria a partir de los cuales se pueden derivar los criterios microbiológicos. A modo de ejemplo, un PO podría establecerse en una proporción del 99.75\% de las unidades de un lote no debe presentar una concentración superior a 4 log ufc/g. Si asumimos que la contaminación del lote sigue una distribución log normal con un valor medio de contaminación $(\mu)$ de 1.75 log ufc/g y una desviación estándar (SD) de 0.8 log ufc/g, se puede derivar un criterio microbiológico igual a $n=7 ; c=0 ; m=2 \log u f c / g$, de forma que haya una probabilidad de rechazo del lote del 95\%.

Sin embargo, en algunas ocasiones dichos criterios microbiológicos no son factibles a la hora de ponerse en práctica por lo que se utilizan fundamentalmente como herramientas para verificar que se han llevado a cabo las medidas de control necesarias para garantizar la seguridad alimentaria.

\section{APLICACIONES INFORMÁTICAS EXISTENTES PARA LA APLICACIÓN DE CRITERIOS MICROBIOLÓGICOS EN ALIMENTOS}

En los últimos años ha surgido el desarrollo de distintas herramientas informáticas de toma de decisiones mediante las cuales se puede evaluar la eficiencia de la implementación de planes de muestreo y criterios microbiológicos. En la opinión publicada por EFSA se resumen los fundamentos y los requisitos principales para el desarrollo de criterios microbiológicos en alimentos (EFSA Panel on Biological Hazards et al., 2017). Las principales aplicaciones informáticas se resumen en la tabla 1.

La herramienta elaborada por la ICMSF consiste en una hoja de Excel que permite evaluar planes de muestreo de dos y tres clases, así como criterios microbiológicos. La versión más reciente (v8) fue publicada en noviembre de 2016 e incluye distintas opciones como la posibilidad de modificar los valores de sensibilidad y especificidad de los métodos de análisis. 
La aplicación web elaborada por FAO/OMS (2016) es una de las más completas actualmente y permite la evaluación de la eficiencia de los criterios microbiológicos utilizando diferentes distribuciones estadísticas. Una de las opciones más destacadas es la posibilidad de realizar un análisis de sensibilidad que permite la optimización de los planes de muestreo y criterios microbiológicos a través de una interfaz sencilla. Asimismo, existen aplicaciones específicas orientadas hacia herramientas de gestión del riesgo y planes de muestreo de Campylobacter spp. y Salmonella spp. en carne de ave, Cronobacter spp. en formulaciones infantiles, histamina y micotoxinas.

La herramienta Baselineapp (v1.0), desarrollada por la Universidad de Córdoba en 2013, constituye un repositorio de modelos predictivos y planes de mues- treo en distintas categorías de alimentos para peligros biológicos específicos. La aplicación web permite calcular y derivar criterios microbiológicos basados en el establecimiento de un FSO o un PO.

Por último, la herramienta TRiMiCri permite desarrollar criterios microbiológicos para Campylobacter spp. en muestras de piel de pollo después del sacrificio. Los criterios microbiológicos están fundamentados en medidas de gestión del riesgo, tales como la reducción del riesgo o (risk reduction) obtenida como consecuencia de la aplicación del criterio, o el cálculo de la proporción del número de lotes no conformes con dicho criterio. La versión actual (1.3), disponible como aplicación de escritorio, está desarrollada por el centro Technical University of Denmark (DTU).

Tabla 1. Resumen de las principales herramientas informáticas existentes para la evaluación de los criterios microbiológicos en alimentos

\begin{tabular}{|c|l|l|}
\hline $\begin{array}{c}\text { Nombre de la } \\
\text { aplicación }\end{array}$ & \multicolumn{1}{|c|}{ Enlace web } & \multicolumn{1}{c|}{ Especificaciones } \\
\hline ICMSF tool & $\begin{array}{l}\text { http://www.icmsf.org/main/software_ } \\
\text { downloads.html }\end{array}$ & $\begin{array}{l}\text { Hoja de Excel donde se pueden aplicar planes de } \\
\text { muestreo por atributos (dos y tres clases), así como } \\
\text { criterios microbiológicos. }\end{array}$ \\
\hline JEMRA tool & $\begin{array}{l}\text { http://www.fstools.org/Sampling/ } \\
\text { SamplingPlans.aspx }\end{array}$ & $\begin{array}{l}\text { Herramienta web que permite evaluar la eficiencia } \\
\text { de los criterios microbiológicos. Incluye aplicaciones } \\
\text { específicas para Campylobacter, Salmonella y } \\
\text { Cronobacter sakazakii }\end{array}$ \\
\hline Baseline tool & http://www.baselineapp.com/ & $\begin{array}{l}\text { Herramienta que combina modelos de microbiología } \\
\text { predictiva y criterios microbiológicos basados en } \\
\text { métricas del riesgo. }\end{array}$ \\
\hline TRiMiCri & http://tools.food.dtu.dk/trimicri & $\begin{array}{l}\text { Aplicación que permite evaluar la eficiencia de la } \\
\text { implementación de criterios microbiológicos para } \\
\text { Campylobacter en carne de ave. }\end{array}$ \\
\hline
\end{tabular}

Fuente: elaboración propia.

\section{BIBLIOGRAFÍA}

Bassett, J., Jackson, T., Jewell, K., Jongenburger, I y Zwietering, M. H. (2010). Impact of Microbial Distributions on Food Safety. International Life Science Institute (ILSI).

EFSA Panel on Biological Hazards (BIOHAZ), Ricci, A., Allende, A., Bolton, D., Chemaly, M., Davies, R., Fernández Escámez, P. S. [...] y Nørrung, B. (2017). Guidance on the development of microbiological criteria. EFSA Journal 15 (11), e05052. https://doi. org/10.2903/j.efsa.2017.5052

International Commission on Microbiological Specifications for Foods (ICMSF) (2002). Microorganisms in Foods
7. Microbiological testing in food safety management. New York: Kluwer Academic/Plenum Publisher.

Schothorst, M. van, Zwietering, M. H., Ross, T., Buchanan, R. L. y Cole, M. B. (2009). Relating microbiological criteria to food safety objectives and performance objectives. Food Control, 20 (11), pp. 967-979. https://doi. org/10.1016/j.foodcont.2008.11.005

Stringer, M. (2005). Summary report. Food Safety Objectives - role in microbiological food safety management. Food Control, 16 (9), pp. 775-794. https://doi.org/10.1016/j. foodcont.2004.10.018
Valero, A. (2015). Predictive tools and strategies for establishing risk-based microbiological criteria in foods. En Sanchís, V., Liébana, E., Romagosa, I. y López Francos, A. (eds.) Food Safety Challenges for Mediterranean Products. Zaragoza: CIHEAM, pp. 67-74.

World Health Organization / Food and Agriculture Organization of the United Nations (2006). Food Safety Risk Analysis: A Guide for National Food Safety Authorities. FAO Food and Nutrition Paper 87. Disponible en https:// apps.who.int/iris/bitstream/handle/10665/43718/9789251056042_ eng.pdf 
World Health Organization / Food and Agriculture Organization of the United Nations (2016). Statistical aspects of microbiological criteria related to foods. A risk managers guide. Microbiological Risk Assessment Series, 24. Disponible en https:// apps.who.int/iris/bitstream/hand le/10665/249531/9789241565318 eng.pdf

Zwietering, M. H., Gorris, L. G. M. y Farber, J. M. (2015). Operationalising a performance objective with a microbiological criterion using a risk-based approach. Food Control, 58, pp. 3342. https://doi.org/10.1016/j.foodcont.2014.07.042

\section{Recursos en línea}

Codex Alimentarius Commission. CAC/GL 50-2004. Directrices generales sobre muestreo. [En línea]. Disponible en: http://www.fao.org/input/download/ standards/10141/CXG_050s.pdf

Codex Alimentarius Commission. CAC/GL 62-2007. Working principles for risk analysis for food safety for application by governments. [En línea]. Disponible en: http://www.fao.org/input/download/standards/10751/CXG_062e.pdf

Commission Regulation (EC) No 178/2002 of the European Parliament and of the Council of 28 January 2002 laying down the general principles and requirements of food law, establishing the European
Food Safety Authority and laying down procedures in matters of food safety. Official Journal of European Union L 31, pp. 1-24. [En línea]. Disponible en: http:// data.europa.eu/eli/reg/2002/178/oj

Commission Regulation (EC) No 852/2004 of the European Parliament and of the Council of 29 April 2004 on the hygiene of foodstuffs. Official Journal of European Union, L226, pp. 3-21. [En línea]. Disponible en: http://data. europa.eu/eli/reg/2004/852/2009-04-20

Commission Regulation (EC) No 1441/2007 of 5 December 2007 amending Regulation (EC) No 2073/2005 on microbiological criteria for foodstuffs. Official Journal of European Union L 322, pp. 12-29. [En línea]. Disponible en: http://data.europa. eu/eli/reg/2007/1441/oj 